\title{
THE SPECTRAL EXTENSION PROPERTY AND EXTENSION OF MULTIPLICATIVE LINEAR FUNCTIONALS
}

\author{
MICHAEL J. MEYER
}

(Communicated by Palle E. T. Jorgensen)

\begin{abstract}
Let $\mathscr{A}$ be a commutative Banach algebra. Denote the spectral radius of an element $a$ in $\mathscr{A}$ by $\rho_{\mathscr{A}}(a)$. An extension of $\mathscr{A}$ is a Banach algebra $\mathscr{B}$ such that $\mathscr{A}$ is algebraically, but not necessarily continuously, embedded in $\mathscr{B}$. We view $\mathscr{A}$ as a subalgebra of $\mathscr{B}$. If $\mathscr{B}$ is an extension of $\mathscr{A}$ then $S p_{\mathscr{B}}(a) \cup\{0\} \subseteq S p_{\mathscr{A}}(a) \cup\{0\}$ and thus $\rho_{\mathscr{B}}(a) \leq \rho_{\mathscr{A}}(a), \forall a \in \mathscr{A}$.

Let us say that $\mathscr{A}$ has the spectral extension property if $\rho_{\mathscr{B}}(a)=\rho_{\mathscr{A}}(a)$ for all $a \in \mathscr{A}$ and all extensions $\mathscr{B}$ of $\mathscr{A}$, that $\mathscr{A}$ has the strong spectral extension property if $S p_{\mathscr{B}}(a) \cup\{0\}=S p_{\mathscr{A}}(a) \cup\{0\}$ for all $a \in \mathscr{A}$ and all extensions $\mathscr{B}$ of $\mathscr{A}$, and that $\mathscr{A}$ has the multiplicative Hahn-Banach property if every multiplicative linear functional $\chi$ on $\mathscr{A}$ has a multiplicative linear extension to every commutative extension $\mathscr{B}$ of $\mathscr{A}$.

We give characterizations of these properties for semisimple commutative Banach algebras.
\end{abstract}

\section{INTRODUCTION}

Let $\mathscr{A}$ be a commutative Banach algebra and denote the spectral radius in $\mathscr{A}$ by $\rho_{\mathscr{A}}$. An extension of $\mathscr{A}$ is a Banach algebra $\mathscr{B}$ such that $\mathscr{A}$ is algebraically, but not necessarily continuously, embedded in $\mathscr{B}$. We view $\mathscr{A}$ as a subalgebra of $\mathscr{B}$. If $\mathscr{B}$ is an extension of $\mathscr{A}$, then $S p_{\mathscr{B}}(a) \cup\{0\} \subseteq$ $S p_{\mathscr{A}}(a) \cup\{0\}$ and thus $\rho_{\mathscr{B}}(a) \leq \rho_{\mathscr{A}}(a)$ for all $a \in \mathscr{A}$.

In 1949 I. Kaplansky [4, Theorem 6.2] showed that for the algebra $\mathscr{A}$ of continuous functions on a compact Hausdorff space, in fact,

$$
\rho_{\mathscr{B}}(a)=\rho_{\mathscr{A}}(a), \quad \forall a \in \mathscr{A},
$$

for every commutative extension and hence for every extension $\mathscr{B}$ of $\mathscr{A}$. C. E. Rickart in [8] extended this result to all semisimple, commutative, completely regular Banach algebras $\mathscr{A}$ and showed that these algebras have the following stronger property:

Every multiplicative linear functional $\chi$ on $\mathscr{A}$ has a (multiplicative linear) extension to every commutative extension $\mathscr{B}$ of $\mathscr{A}$.

Received by the editors January 26, 1990; presented at the Regional Meeting of the AMS, Special Session on Banach Algebras, Fayetteville, Arkansas, March 24, 1990.

1980 Mathematics Subject Classification (1985 Revision). Primary 46J15, 46J05, 47D15. 
From this (1) immediately follows. Here the following intermediate property is also of interest:

$$
S p_{\mathscr{B}}(a) \cup\{0\}=S p_{\mathscr{A}}(a) \cup\{0\},
$$

for all $a \in \mathscr{A}$ and all extensions $\mathscr{B}$ of $\mathscr{A}$.

We say that $\mathscr{A}$ has the spectral extension property if it satisfies (1), the multiplicative Hahn-Banach property if it satisfies (2), and the strong spectral extension property if it satisfies (3). It is not difficult to see that the multiplicative Hahn-Banach property implies the strong spectral extension property, that in turn applies the spectral extension property.

We characterize the semisimple commutative Banach algebras having each property by a condition similar to, but weaker than, complete regularity of $\mathscr{A}$, and increasing "size" of the Shilov boundary $\partial \mathscr{A}$ of $\mathscr{A}$ in the entire carrier space $\Gamma(\mathscr{A})$ of $\mathscr{A}$, in a sense that is clarified below.

As a result, we can give examples of Banach algebras with the spectral extension property and the multiplicative Hahn-Banach property that are not completely regular. It also follows that a semisimple infinite-dimensional Banach algebra with the spectral extension property contains zero divisors.

For an element $a \in \mathscr{A}$, introduce the permanent radius $\rho_{p}(a):=\inf _{\mathscr{B}} \rho_{\mathscr{B}}(a)$, where the infimum is taken over all extensions $\mathscr{B}$ of $\mathscr{A}$. Call a subset $F$ of the carrier space $\Gamma(A)$ of $\mathscr{A}$ a set of uniqueness, if its kernel in $\mathscr{A}$ is trivial; equivalently, if $\hat{a}=0$ on $F \Rightarrow a=0$ for all elements $a \in \mathscr{A}$. If $\mathscr{A}$ is semisimple, then every set containing the Shilov boundary is a set of uniqueness, but in general there may be closed sets of uniqueness that do not contain the Shilov boundary (see, for example, the disc algebra). Our main results can be stated as follows:

Theorem 1. For a semisimple commutative Banach algebra $\mathscr{A}$, the following are equivalent:

(1) A has the spectral extension property.

(2) Every closed set of uniqueness $F \subseteq \Gamma(\mathscr{A})$ contains the Shilov boundary.

(3) If $F \subseteq \Gamma(\mathscr{A})$ is a closed subset that does not contain the Shilov boundary of $\mathscr{A}$, then there exists an element $a \in \mathscr{A}$ such that $\hat{a}=0$ on $F$ and $\rho_{p}(a)>0$.

(4) Every multiplicative linear functional $\chi$ in the Shilov boundary of $\mathscr{A}$ has a (multiplicative, linear) extension to every commutative extension $\mathscr{B}$ of $\mathscr{A}$.

Theorem 2. Let $\mathscr{A}$ be a semisimple commutative Banach algebra. Then $\mathscr{A}$ has the strong spectral extension property if and only if it has the spectral extension property and the Shilov boundary $\partial \mathscr{A}$ of $\mathscr{A}$ satisfies

$$
\hat{a}(\partial \mathscr{A}) \cup\{0\}=\hat{a}(\Gamma(\mathscr{A})) \cup\{0\}, \quad \forall a \in \mathscr{A} .
$$

Theorem 3. Let $\mathscr{A}$ be a semisimple commutative Banach algebra. Then $\mathscr{A}$ has the multiplicative Hahn-Banach property if and only if it has the spectral 
extension property and the Shilov boundary $\partial \mathscr{A}$ is the entire carrier space of $\mathscr{A}$.

\section{Proofs}

Suppose that $\mathscr{A}$ is a commutative Banach algebra. Denote by $\bar{\Gamma}(\mathscr{A})$ the space of all multiplicative linear functionals on $\mathscr{A}$ including the zero functional. Then $\bar{\Gamma}(\mathscr{A})$ is always a compact Hausdorff space in the topology of pointwise convergence on $\mathscr{A}$ ( $\mathscr{A}$-topology) even if $\mathscr{A}$ does not have an identity. Also $\bar{\Gamma}(\mathscr{A})=\Gamma(\mathscr{A}) \cup\{0\}$, where $\Gamma(\mathscr{A})$ denotes the carrier space of $\mathscr{A}$.

For $a \in \mathscr{A}$ let $\hat{a} \in \mathscr{C}(\bar{\Gamma}(\mathscr{A}))$ denote the Gelfand transform of $a$. For a subset $F \subseteq \bar{\Gamma}(\mathscr{A})$ and an element $a \in \mathscr{A}$, let

$$
\|\hat{a}\|_{F}:=\sup _{\chi \in F}|\hat{a}(\chi)|=\sup _{\chi \in F}|\chi(a)| \quad \text { and } \quad\|\hat{a}\|_{\infty}:=\|\hat{a}\|_{\bar{\Gamma}(\mathscr{A})} .
$$

Recall that $S p_{\mathscr{A}}(a) \cup\{0\}=\hat{a}(\Gamma(\mathscr{A})) \cup\{0\}$, thus $\|\hat{a}\|_{\infty}=\rho_{\mathscr{A}}(a)$, and that $\hat{a}$ is a continuous function vanishing at infinity on the locally compact Hausdorff space $\Gamma(\mathscr{A})$. Thus $|\hat{a}|$ assumes its maximum on every closed subset $F \subseteq$ $\Gamma(\mathscr{A})$. Recall also that the Shilov boundary $\partial \mathscr{A}$ of $\mathscr{A}$ is the smallest closed subset $F \subseteq \Gamma(\mathscr{A})$ such that $\|\hat{a}\|_{\infty}=\|\hat{a}\|_{F}$ for all $a \in \mathscr{A}$.

In particular, then, for $a \in \mathscr{A},|\hat{a}|$ assumes its maximum on $\partial \mathscr{A}$. Thus we can choose a multiplicative linear functional $\chi \in \partial \mathscr{A}$ such that $|\chi(a)|=\rho_{\mathscr{A}}(a)$ (see $[7,3.1])$.

Proposition 1. The following are equivalent for a commutative Banach algebra $\mathscr{A}:$

(1) $\mathscr{A}$ has the spectral extension property.

(2) Every submultiplicative norm \|\| on $\mathscr{A}$ and every $a \in \mathscr{A}$ satisfy $\rho(a) \leq\|a\|$.

(3) Every extension $\mathscr{B}$ of $\mathscr{A}$ and every $a \in \mathscr{A}$ satisfy $\partial\left(S p_{\mathscr{A}}(a)\right) \subseteq$ $S p_{\mathscr{B}}(a) \cup\{0\}$.

Proof [6]. We will only use the equivalence of (1) and (2) and include the short argument: If $\mathscr{B}$ is an extension of $\mathscr{A}$ and \|\| the complete norm on $\mathscr{B}$, then for any element $a \in \mathscr{A}$ :

$$
\begin{aligned}
\rho_{\mathscr{B}}(a) & =\rho_{\mathscr{A}}(a) \Leftrightarrow \rho_{\mathscr{A}}(a) \leq \rho_{\mathscr{B}}(a) \Leftrightarrow \\
\rho_{\mathscr{A}}\left(a^{n}\right) & =\rho_{\mathscr{A}}(a)^{n} \leq\left\|a^{n}\right\| \quad \text { for all } n \geq 1 .
\end{aligned}
$$

Definition 1. For an element $a \in \mathscr{A}$, define the permanent radius $\rho_{p}(a)$ by $\rho_{p}(a):=\inf _{\mathscr{B}} \rho_{\mathscr{B}}(a)$, where the infimum is taken over all extensions $\mathscr{B}$ of $\mathscr{A}$.

Clearly $\rho_{p}(a) \leq \rho_{\mathscr{A}}(a)$. From [2, I.4.2] it follows immediately that $\rho_{p}(a):=$ $\inf \|a\|$, where the infimum is taken over all submultiplicative norms \|\| on $\mathscr{A}$. We will be interested in elements $a \in \mathscr{A}$ that satisfy $\rho_{p}(a)>0$. 
Proposition 2. Let $\mathscr{A}$ be any Banach algebra (not necessarily commutative).

(1) If $a \in \mathscr{A}$ is a quasidivisor of zero (ax $=x$ or $x a=x$ for some nonzero $x \in \mathscr{A})$ then $\rho_{p}(a) \geq 1$.

(2) If $a, h \in \mathscr{A}$ are such that ah $=$ ha is a quasidivisor of zero then $\rho_{p}(a) \geq 1 / \rho_{\mathscr{A}}(h)$.

(3) $\rho_{p}(a) \geq \operatorname{dist}(C, 0)$, where $C$ is any connected component of $S p_{\mathscr{A}}(a)$.

Proof. (1) Obvious.

(2) For every extension $\mathscr{B}$ of $\mathscr{A}$ we have $1 \leq \rho_{\mathscr{B}}(a h) \leq \rho_{\mathscr{B}}(a) \rho_{\mathscr{B}}(h) \leq$ $\rho_{\mathscr{B}}(a) \rho_{\mathscr{A}}(h)$.

(3) We may assume that $r:=\operatorname{dist}(C, 0)>0$. Let $s<r$ be arbitrary. $S p(a)$ is a compact subset of the complex plane. According to [5, Corollary 1 , p. 83] we can find a nonempty open and closed subset $F \subseteq S p(a)$ such that $\operatorname{dist}(\lambda, S p(a))<r-s$ for all $\lambda \in F$ (see also [1]). In particular then, $\operatorname{dist}(0, F)>s$. Choose disjoint open sets $U, V$ in the plane such that $0 \notin$ $U, F \subseteq U$, and $S p(a) \backslash F \subseteq V$. Let $f(\lambda)$ be the holomorphic function on $U \cup V$ that satisfies $\lambda f(\lambda)=1$ on $U$ and $f=0$ on $V$. By the holomorphic functional calculus, the element $a f[a]=f[a] a \in \mathscr{A}$ is an idempotent. Since $F$ is nonempty, $1 \in S p(a f[a])$, by the Spectral Mapping Theorem, and so $a f[a] \neq$ 0 . It follows that $a f[a]$ is a quasidivisor of zero. Notice that $\rho(f[a]) \leq 1 / s$, again by the Spectral Mapping Theorem. It follows from (2) that $\rho_{p}(a) \geq$ $1 / \rho(f[a]) \geq s$.

Proof of Theorem 1. (1) $\Rightarrow(2)$. Let $F \subseteq \Gamma(\mathscr{A})$ be a closed set of uniqueness. Then $\|a\|:=\|\hat{a}\|_{F}, \quad \forall a \in \mathscr{A}$, defines a submultiplicative norm on $\mathscr{A}$. Since $\mathscr{A}$ has the spectral extension property, we have $\|\hat{a}\|_{F}=\|a\| \geq \rho_{\mathscr{A}}(a)=\|\hat{a}\|_{\infty}$, $\forall a \in \mathscr{A}$. It follows that $\partial \mathscr{A} \subseteq F$.

$(2) \Rightarrow(3)$. Notice first that we have $\rho_{p}(a)=\rho_{\mathscr{A}}(a)>0$ for every nonzero $a \in \mathscr{A}$, since $\mathscr{A}$ has the spectral extension property and is semisimple.

Now let $F \subseteq \Gamma(\mathscr{A})$ be a closed subset that does not contain the Shilov boundary $\partial \mathscr{A}$ of $\mathscr{A}$. Then $F$ is not a set of uniqueness, and so there exists a nonzero element $a \in \mathscr{A}$ such that $\hat{a}=0$ on $F$.

(3) $\Rightarrow(4)$. Let $\mathscr{B}$ be a commutative extension of $\mathscr{A}$. Since pointwise convergence on $\mathscr{B}$ implies pointwise convergence on $\mathscr{A}$, the restriction map:

$$
\phi: \chi \in \bar{\Gamma}(\mathscr{B}) \rightarrow \chi_{\mid A} \in \bar{\Gamma}(\mathscr{A})
$$

is continuous, and hence $\phi(\bar{\Gamma}(\mathscr{B})) \subseteq \bar{\Gamma}(\mathscr{A})$ is compact and therefore closed. Then $F:=\phi(\bar{\Gamma}(\mathscr{B})) \cap \Gamma(\mathscr{A})$ is a closed subset of $\Gamma(\mathscr{A})$ and we wish to show that $\partial \mathscr{A} \subseteq F$. Notice that for all $a \in \mathscr{A}$,

$$
\rho_{\mathscr{B}}(a)=\|\hat{a}\|_{\Gamma(\mathscr{B})}=\|\hat{a}\|_{F},
$$

since $\phi(\Gamma(\mathscr{B})) \backslash F$ consists only of functionals $\chi$ on $\mathscr{B}$ for which $\hat{a}(\chi)=0$, $\forall a \in \mathscr{A}$. Assume now that $F$ does not contain the Shilov boundary of $\mathscr{A}$. Then we can choose an element $a \in \mathscr{A}$ such that $\rho_{p}(a)>0$ and $\hat{a}=0$ on $F$. 
But then we have the contradiction:

$$
\rho_{p}(a) \leq \rho_{\mathscr{B}}(a)=\|\hat{a}\|_{F}=0 .
$$

(4) $\Rightarrow(1)$. Let $\mathscr{B}$ be an extension of $\mathscr{A}$ and $a \in \mathscr{A}$. We wish to show that $\rho_{\mathscr{B}}(a)=\rho_{\mathscr{A}}(a)$. We may assume that $\mathscr{B}$ is commutative (otherwise replace $\mathscr{B}$ with a maximal commutative subalgebra containing $\mathscr{A}$ ). Choose a multiplicative linear functional $\chi \in \partial \mathscr{A}$ such that $|\chi(a)|=\rho_{\mathscr{A}}(a)$. Then $\chi$ extends to a multiplicative linear functional on $\mathscr{B}$ and therefore

$$
\rho_{\mathscr{A}}(a)=|\chi(a)| \leq \rho_{\mathscr{B}}(a) \leq \rho_{\mathscr{A}}(a) \text {. }
$$

Remark. G. Shilov has shown in [9] that every $\chi \in \partial \mathscr{A}$ has an extension to every isometric extension $\mathscr{B}$ of $\mathscr{A}$. The same argument shows that all $\chi \in \partial \mathscr{A}$ extend to $\mathscr{B}$ whenever $\rho_{\mathscr{B}}(a)=\rho_{\mathscr{A}}(a), \forall a \in \mathscr{A}$ (see also [3]).

Corollary 1. A semisimple, infinite-dimensional, commutative Banach algebra $\mathscr{A}$, that has the spectral extension property, contains zero divisors.

Proof. If $F \subseteq \partial \mathscr{A}$ is a proper closed subset, then by Theorem 1 we can choose an element $a \in \mathscr{A}$ such that $\hat{a}=0$ on $F$. Recall now [7, 3.3.3] that the Shilov boundary $\partial \mathscr{A}$ is an infinite Hausdorff space. We may therefore choose two disjoint (relatively) open subsets $U, V \subseteq \partial \mathscr{A}$. Let $F:=\partial \mathscr{A} \backslash U, K:=$ $\partial \mathscr{A} \backslash V$. Choose nonzero elements $a, b \in \mathscr{A}$ such that $\hat{a}=0$ on $F$ and $\hat{b}=0$ on $K$. Then $\widehat{a b}=\hat{a} \hat{b}=0$ on all of $\partial \mathscr{A}$ and hence on all of $\Gamma(\mathscr{A})$. By semisimplicity of $\mathscr{A}$ it follows that $a b=0$.

Lemma 1. Assume that $\mathscr{A}$ is a semisimple commutative Banach algebra. Let $\mathscr{B}=\mathscr{C}_{0}(\partial \mathscr{A})$ be the algebra of all continuous complex valued functions on the Shilov boundary $\partial \mathscr{A}$ of $\mathscr{A}$ that vanish at infinity. Then $\mathscr{B}$ is an extension of $\mathscr{A}$, and if $\chi$ is a nonzero multiplicative linear functional on $\mathscr{A}$ that extends to $\mathscr{B}$, then $\chi \in \partial \mathscr{A}$.

Proof. By semisimplicity of $\mathscr{A}$ the map $a \in \mathscr{A} \rightarrow \hat{a}_{\mid \partial \mathscr{A}} \in \mathscr{B}$ is a 1-1 homomorphism. Thus the $C^{*}$-algebra $\mathscr{B}=\mathscr{C}_{0}(\partial \mathscr{A})$ is an extension of $\mathscr{A}$. The Shilov boundary $\partial \mathscr{A}$ is a locally compact Hausdorff space and it is well known that every nonzero multiplicative linear functional $\mu$ on $\mathscr{B}$ has the form $\mu(f)=f(\chi) \quad \forall f \in \mathscr{B}$, for some $\chi \in \partial \mathscr{A}$. In particular, its restriction to $\mathscr{A}$ satisfies

Thus $\mu_{1 \mathscr{A}}=\chi \in \partial \mathscr{A}$.

$$
\mu_{\mid \mathscr{A}}(a)=\mu(\hat{a})=\hat{a}(\chi)=\chi(a) .
$$

Proof of Theorem 2. Assume first that $\mathscr{A}$ has the strong spectral extension property. Then $\mathscr{A}$ has the spectral extension property. Also, by Lemma 1, the algebra $\mathscr{B}=\mathscr{C}_{0}(\partial \mathscr{A})$ of all continuous complex valued functions on the Shilov boundary $\partial \mathscr{A}$ of $\mathscr{A}$ that vanish at infinity, is an extension of $\mathscr{A}$. Every element $f \in \mathscr{B}$ satisfies $S p_{\mathscr{B}}(f) \cup\{0\}=f(\partial \mathscr{A}) \cup\{0\}$. Thus every element $a \in \mathscr{A}$ satisfies

$$
\hat{a}(\partial \mathscr{A}) \cup\{0\}=S p_{\mathscr{B}}(a) \cup\{0\}=S p_{\mathscr{A}}(a) \cup\{0\}=\hat{a}(\Gamma(\mathscr{A})) \cup\{0\} .
$$


Now assume conversely that $\mathscr{A}$ has the spectral extension property and satisfies $\hat{a}(\partial \mathscr{A}) \cup\{0\}=\hat{a}(\Gamma(\mathscr{A})) \cup\{0\}, \forall a \in \mathscr{A}$. Let $\mathscr{B}$ be any commutative extension of $\mathscr{A}$ and $a \in \mathscr{A}$. If $\lambda \in S p_{\mathscr{A}}(a)$ is nonzero, there exists a multiplicative linear functional $\chi \in \Gamma(\mathscr{A})$ such that $\lambda=\hat{a}(\chi)$. By assumption $\chi$ can be chosen to be in the Shilov boundary $\partial \mathscr{A}$ of $\mathscr{A}$. Since $\mathscr{A}$ has the spectral extension property, $\chi$ extends to $\mathscr{B}$, by Theorem 1. It follows that $\lambda=\hat{a}(\chi) \in S p_{\mathscr{B}}(a)$. This shows that $S p_{\mathscr{A}}(a) \cup\{0\} \subseteq S p_{\mathscr{B}}(a) \cup\{0\}$ and hence that equality holds. It follows that this is true for any, not necessarily commutative, extension $\mathscr{B}$ of $\mathscr{A}$.

Proof of Theorem 3. (2) $\Rightarrow(1)$. This follows immediately from Theorem 1.

(1) $\Rightarrow(2)$. Assume that $\mathscr{A}$ has the multiplicative Hahn-Banach property. Then we have $\partial \mathscr{A}=\Gamma(\mathscr{A})$ in view of Lemma 1 . Now let $\mathscr{B}$ be an extension of $\mathscr{A}$ and $a \in \mathscr{A}$. We wish to show that $\rho_{\mathscr{B}}(a)=\rho_{\mathscr{A}}(a)$. Choose a multiplicative linear functional $\chi$ on $\mathscr{A}$ such that $|\chi(a)|=\rho_{\mathscr{A}}(a)$. Then $\chi$ has an extension to $\mathscr{B}$ and consequently $\rho_{\mathscr{B}}(a) \geq|\chi(a)|=\rho_{\mathscr{A}}(a)$.

Corollary 2 [Rickart, 8]. Every semisimple, completely regular, commutative Banach algebra $\mathscr{A}$ has the multiplicative Hahn-Banach property.

Proof. Clearly complete regularity of $\mathscr{A}$ implies that $\partial \mathscr{A}=\Gamma(\mathscr{A})$. Suppose now that $F \subseteq \Gamma(\mathscr{A})$ is any proper closed subset. Then there exists $a \in \mathscr{A}$ such that $\hat{a}=0$ on $F$ and $\hat{a}=1$ on some nonvoid open subset $U \subseteq \Gamma(\mathscr{A})$. Furthermore, $\mathscr{A}$ contains a nonzero element $b$ such that $\hat{b}=0$ outside $U$. But then $\widehat{a b}=\hat{a} \hat{b}=\hat{b}$ everywhere and hence $a b=b$ by semisimplicity of $\mathscr{A}$. It follows that $\rho_{p}(a) \geq 1$.

Example 1 (Bruce Barnes, personal communication). The following Banach algebra is semisimple and has the multiplicative Hahn-Banach property without being completely regular. Let $D:=\{z:|z|<1\} \subseteq C$ be the open unit disc, $\bar{D}$ the closed unit disc, and $\Phi:=\bar{D} \times[0,1]$. Let $\mathscr{A}$ be the algebra of all continuous functions on $\Phi$ that are holomorphic on $D \times\{0\}$ endowed with the uniform norm. Clearly, $\mathscr{A}$ is semisimple. Notice that $\rho_{\mathscr{A}}(f)=\|f\|_{\infty}$, $\forall f \in \mathscr{A}$. It can be shown that the carrier space $\Gamma(\mathscr{A})$ of $\mathscr{A}$ can be identified with the set $\Phi$ in such a way that for each element $f \in \mathscr{A}$, the Gelfand transform $\hat{f}$ coincides with the function $f$ on $\Phi$.

Clearly $\mathscr{A}$ does not separate points from closed subsets in $D \times\{0\}$. This shows that $\mathscr{A}$ is not completely regular. Notice that $\mathscr{A}$ contains all continuous functions on $\Phi$ that vanish on $D \times\{0\}$. This shows that $\partial \mathscr{A}=\Gamma(\mathscr{A})$.

Now let $F \subseteq \Phi$ be a proper closed subset. Then $\mathscr{A}$ contains a function $f$ that vanishes on $F$ but is identically 1 on an open subset $U \subseteq \Phi$. Also, $\mathscr{A}$ contains a nonzero function $g$ with support in $U$. Then $f g=g$ and this shows that $\rho_{p}(f) \geq 1$ (Proposition 1). By Theorem 1, $\mathscr{A}$ has the spectral extension property. Now it follows from Theorem 3 that $\mathscr{A}$ has the multiplicative Hahn-Banach property. 
Example 2. Suppose that $0<r<R$ and let $\mathscr{A}$ be the algebra of all functions that are continuous on the closed disc $\bar{B}(0, R)$ and holomorphic on the open disc $B(0, r)$, endowed with the uniform norm. It can be shown that the structure space $\Gamma(\mathscr{A})$ can be identified with the disc $\bar{B}(0, R)$ in such a way that the Gelfand transforms $\hat{f}$ coincide with the functions $f \in \mathscr{A}$.

The Shilov boundary $\partial \mathscr{A}$ is the annulus $\{\lambda|r \leq| \lambda \mid \leq R\}$. The function $f(\lambda):=\lambda$ does not take all its values on the Shilov boundary, and so $\mathscr{A}$ does not have the strong spectral extension property. However $\mathscr{A}$ contains all continuous functions on $\bar{B}(0, R)$ that vanish on $\bar{B}(0, r)$. Using Theorem 1 , it can be seen, as in the previous example, that $\mathscr{A}$ has the spectral extension property.

The author does not know of an example of a commutative semisimple Banach algebra with the strong spectral extension property for which the multiplicative Hahn-Banach property fails. Question: For a commutative semisimple Banach algebra $\mathscr{A}$ does the strong spectral extension property imply the multiplicative Hahn-Banach property?

\section{REFERENCES}

1. B. Barnes, Interpolation of spectrum of bounded operators on Lebesgue spaces, Rocky Mountain J. Math. 20 (1990), 359-378.

2. F. F. Bonsall and J. Duncan, Complete normed algebras, Springer-Verlag, New York, 1973.

3. M. Gelfand, D. A. Raikov, and G. E. Shilov, Commutative normed rings, Uspekhi Mat. Nauk. 1 (1946), 48-146.

4. I. Kaplansky, Normed algebras, Duke Math. J. 16 (1949), 399-418.

5. M. Newman, Elements of the topology of plane sets of points, Cambridge Univ. Press, 1954.

6. T. W. Palmer, Spectral algebras, subalgebras and pseudonorms, Rocky Mountain J. Math. (to appear).

7. C. E. Rickart, General theory of Banach algebras, Van Nostrand, New York, 1960.

8. __ On spectral permanence for certain Banach algebras, Proc. Amer. Math. Soc. 4 (1953), 191-196.

9. G. E. Shilov, On the extension of maximal ideals, Dokl. Akad. Nauk. SSSR 29 (1940), 83-84.

Department of Mathematics and Computer Science, Georgia State University, Atlanta, Georgia 30303 